\title{
Negative differential resistance behavior of silicon monatomic chain encapsulated in carbon nanotubes
}

\author{
Yingying Zhang, Feng-Chao Wang, Ya-Pu Zhao* \\ State Key Laboratory of Nonlinear Mechanics, Institute of Mechanics, Chinese Academy of Sciences, Beijing 100190, China
}

\section{A R T I C L E I N F O}

\section{Article history:}

Received 2 October 2011

Received in revised form 28 April 2012

Accepted 30 April 2012

Available online 4 June 2012

\section{Keywords:}

Silicon monatomic chain

Size-selective encapsulation

Electronic transport properties

Negative differential resistance

Transmission spectrum

\begin{abstract}
A B S T R A C T
Using nonequilibrium Green's functions in combination with density-functional theory (DFT), we investigated the electronic transport properties of the silicon monatomic chains (SiMCs) with different geometries which were induced by the encapsulation of the carbon nanotubes (CNTs). The encapsulated SiMCs, which were put inside $(5,5),(6,6),(7,7)$ and $(8,8)$ hydrogenated armchair CNTs, were coupled to two Au $\left(\begin{array}{lll}1 & 0 & 0\end{array}\right)$ nanoscale electrodes. The electronic transport property of an isolated finite SiMC was also studied to serve as a reference to our calculations. As the diameter of CNTs increases, the geometry structures of SiMCs changed. Calculated results show that the current-voltage $(I-V)$ characteristics depend sensitively on the geometry structures of SiMCs and can be controlled by the size-selective encapsulation. Negative differential resistance (NDR) phenomena were observed within certain bias voltage ranges. A detailed analysis of the origin of NDR was carried out with the transmission spectrum, the spatial distribution of frontier molecular orbitals and the molecular projected self-consistent Hamiltonian (MPSH) states taken into consideration. These results indicated that the size-selective encapsulation of SiMCs in CNTs can become a possible candidate for designing the silicon-based nanoelectronic devices.
\end{abstract}

() 2012 Elsevier B.V. All rights reserved.

\section{Introduction}

One dimensional (1-D) nanomaterials, such as nanowires, nanorods, and carbon nanotubes (CNTs), are regarded as promising building blocks for future miniature devices because of their unique structures and small dimensions $[1,2]$. Silicon nanowires have recently been utilized in many device applications such as thermoelectric materials [3], solar cells [4], sensors [5], as well as field-effect transistor [6], attributing to the rapid development of synthetic and fabrication technologies. Silicon monatomic chain (SiMC) is the ultimate silicon nanowire and provides a promising concept for the bottom-up approach to nanoelectronics. The electron transport properties of SiMCs have been studied theoretically [7] and may provide us the ultimate miniaturization and novel functionality of electronic devices at nanoscale. CNTs have attracted interest not only for their remarkable properties, such as high mechanical strength [8], high thermal and chemical stabilities [9], excellent heat conduction [10], and interesting electrical and electronic properties [11] but also because their hollow interior can serve as a nanometer-sized mold or template in material fabrication. Recently, significant interest has been shown on the filling of CNTs with materials and various investigations were carried out by researchers, such as the study of the transfer characteristics of

\footnotetext{
* Corresponding author. Tel.: +86 10 82543932; fax: +86 1082543977 .

E-mail address: yzhao@imech.ac.cn (Y.-P. Zhao).
}

water confined in CNTs [12], the structural transformation of partially confined copper nanowires inside defected CNTs [13], the structures and electrical transport properties of germanium nanowires encapsulated in CNTs [14], the structural, electronic, and vibrational properties of the finite carbon chains encapsulated inside CNTs [15], etc. These studies have revealed that matter in a confined nanospace might exhibit extraordinary unusual structures and electronic properties, which is quite different from the behavior of their bulk counterparts.

It should be noted that the similar monatomic chains were reported only in a few experiments [16] or modeled in simulations $[17,18]$. However, in recent years, research on the electron transport properties of molecular devices has attracted increasing attention. On the nanoscale, many interesting physical transport properties, such as single electron characteristic [19], negative differential resistance (NDR) [20-22], molecular rectification [23] and field-effect characteristics [24] have been found through the molecular devices. The most prominent effect among these is the NDR behavior. The NDR phenomenon in molecular conductors, which is characterized by a decreasing current through the junction at an increasing voltage bias, has gained widespread interest because of its wide range of applications in electronic devices including amplification, logic and memory, as well as fast switching. Some groups experimentally and theoretically observed NDR in many physical systems such as oligo (phenylene ethynylene) (OPE) molecular devices with semiconductor electrodes [20], 
doped and squashed $\mathrm{C}_{60}$ molecular device [21], porphyry molecular junctions modulated with side groups, CNTs or heterojunctions with the metal electrodes [22]. Possible mechanisms have been proposed to explain the NDR behavior, such as the variation of the coupling between the molecular orbital and the incident states from the electrodes at different bias voltages [20], the channel conduction being suppressed at a certain bias [22], the resonant and off resonant electronic tunneling mechanism [25], the biasinduced alignment of molecular orbitals [26], side group effects [27], and the splitting of the molecular orbitals [28]. In spite of a number of studies about NDR in various kinds of molecular devices, the origin for NDR is still under intense debate due to their structural complexity.

SiMC and CNTs serve as molecularly perfect materials and have opened up opportunities for the design of nano-electronic devices [29]. As the scale of microelectronic engineering continues to shrink, interest has focused on the electron transport through essentially one-dimensional nanometre-scale channels such as atomic chain. Understanding electron transport in the ultimate limit of devices of the atomic chain is challenging, but experimental studies have reported the electrical transport property of individual rows of suspended gold atoms and CNT-clamped metal atomic chain $[16,30]$. $\mathrm{Ng}$ and co-workers have showed that the transport characteristics of silicon nanowires depend strongly on their geometry [31]. Fan et al. [15] and Rusznyak et al. [32] have found that structure of monatomic chain changes when encapsulated in CNTs. Li et al. [14,33] investigated the structures and electrical transport properties of germanium and nickel nanowires encapsulated in CNTs. Motivated by experimental works $[16,30]$ and considered recent theoretical studies [14,33,34], the electronic transport properties of SiMCs with different geometries which were induced by the encapsulation of different CNTs were investigated by applying density-functional theory (DFT) combined with nonequilibrium Green's functions (NEGF). In this work, the NDR phenomena of the SiMCs were determined with the effects of the encapsulation of CNTs taken into consideration. In order to explain the electronic transport properties of SiMCs, we investigated the transmission spectrum and molecular projected self-consistent Hamiltonian (MPSH) of the frontier molecular orbitals under various voltages. These results indicate that the size-selective encapsulation of SiMCs in CNTs can become a possible candidate for designing the silicon-based nanoelectronic devices.

\section{Model and calculation method}

The geometry of an isolated SiMC was calculated to serve as a reference to our calculations. Therefore, both the isolated SiMC and the encapsulated SiMCs in hydrogenated CNTs $(5,5),(6,6)$, $(7,7)$ and $(8,8)$ were considered in this work. Geometry optimizations of these systems were performed to obtain the stable configurations using the Dmol ${ }^{3}$ modeling software packages and the results were shown in Fig. 1. Calculations were based on DFT with the electronic exchange and correlation effects described by the generalized gradient approximation (GGA). Atomic coordinates were adjusted without any symmetry constraint with a convergence criterion of $0.0027 \mathrm{eV}$ for the energy and $0.05 \AA$ for the displacement. For the SiMC encapsulated in the $(5,5) \mathrm{CNT}$, significant bond length changes can be observed compared with the isolated SiMC. The SiMCs in the $(6,6)$ and $(7,7)$ CNTs presented the bond angle alternations shown in Fig. 1c and d. These changes can be interpreted by a combined effect of hybridization and charge transfer between the chain and the tube. The chain-tube interaction decreases with increasing diameter of the tube. When the SiMC was encapsulated inside the $(8,8) \mathrm{CNT}$, its geometry was almost the same as the isolated SiMC due to the weaker chain-tube interaction because of the larger diameter.
Since the geometry structures of the encapsulated SiMCs have been determined in the optimization calculations as illustrated in Fig. 1, here we constructed a series of the two-probe systems to study the effect of the encapsulation on the electronic transport properties of the SiMCs. These systems, which were denoted as M1-M5, are shown in Fig. 2. The CNTs were just omitted for the simplification, since the geometry structures shown in Fig. 2 were obtained under the effect of the encapsulation. The transport calculations were performed using the ATK software package [35], which is based on real-space NEGF technique and DFT. Each SiMC was connected with two parallel Au ( $\left.\begin{array}{lll}1 & 0 & 0\end{array}\right)$ surfaces corresponding to the surfaces of gold electrodes. Each layer of the electrode was represented by a $4 \times 4$ supercell with the periodic boundary conditions so that it can be used to imitate bulk metal structures. The supercell had a large enough vacuum layer in the $x$ and $y$ directions so that there is little interaction between the SiMC and its mirror images and $z$ is the transport direction. During the calculations, the system consisting of the SiMC and two gold electrodes was divided into three regions, namely, the left electrode, the scattering region and the right electrode. The SiMC together with three surface atomic layers in the left electrode and two surface atomic layers in the right electrode was chosen as the central scattering region, as indicated in Fig. 2. The first atom of the SiMC was positioned symmetrically above the Au ( $\left.\begin{array}{lll}1 & 0 & 0\end{array}\right)$ hollow sites while the last atom position was determined by the SiMC geometry. The contact distance between the SiMC and the electrodes was fixed at $2.56 \AA$ A. In our transport calculations, a single-zeta plus polarization basis set was employed to describe the localized atomic orbitals and an energy cutoff for real-space mesh size was set to be 150 Ry to get a reasonable balance between calculation efficiency and accuracy. A $5 \times 5 \times 100 \mathrm{k}$-point mesh in the $x, y$, and $z$ was sampled in the Brillion Zone according to the Monkhorst-Pack method. The current was calculated from the Landauer formula,

$I(V)=\frac{2 e^{2}}{h} \int_{\mu_{L}}^{\mu_{R}} T\left(E, V_{b}\right) d E$,

where $e$ is the elementary charge, $h$ is the Planck constant, and the constant $2 e^{2} / h$ is the conductance quantum. $\mu_{L}$ and $\mu_{R}$ are the electrochemical potentials of the left and right electrodes, respectively. The total transmission probability $T\left(E, V_{b}\right)=\sum_{n} T_{n}\left(E, V_{b}\right)$ for electrons incident at an energy $E$ through the device under the potential bias voltage $V_{b}$ is composed of all available conduction channels with the individual transmission $T_{n} .\left[\mu_{\mathrm{L}}, \mu_{R}\right]$ is the energy region which contributes to the current integral and is referred to as the bias window. Considering the fact that the average Fermi level $E_{F}$ is set to be zero, the bias window is actually $[-V / 2, V / 2]$

\section{Results and discussion}

\subsection{I-V characteristic}

Fig. 3 plotted the calculated currents as a function of the applied bias voltage varying from 0 to $4 \mathrm{~V}$ for the system of M1-M5. Evolutions of the currents clearly show that the transport properties of each system are dependent sensitively on the SiMC geometric structure which is modulated by the CNT encapsulation. The $I-V$ curves of M1 and M5 are almost the same because of the similar geometries of the two systems. For M2, M3 and M4, the currents are smaller than that of M1 and M5 when the applied voltage was lower than $2.2 \mathrm{~V}$, which is resulted from the bond length or bond angle changes of SiMCs due to the chain-tube interaction. When the applied voltage is larger than $2.2 \mathrm{~V}$, the NDR phenomenon can be observed for all the systems M1-M5. When the applied bias voltage is larger than $3.0 \mathrm{~V}$, the currents for these systems can be ranked as $I_{\mathrm{M} 4}>I_{\mathrm{M} 3}>I_{\mathrm{M} 2}>I_{\mathrm{M} 5}>I_{\mathrm{M} 1}$ at the same bias voltage and another NDR behavior can be observed for all the five systems. The 


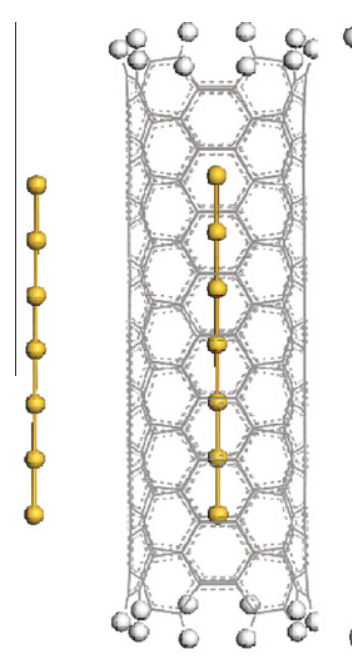

(a)

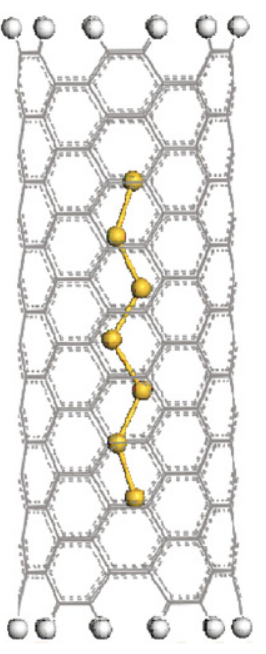

(c)

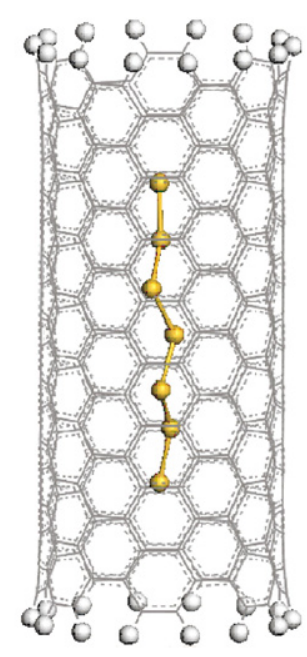

(d)

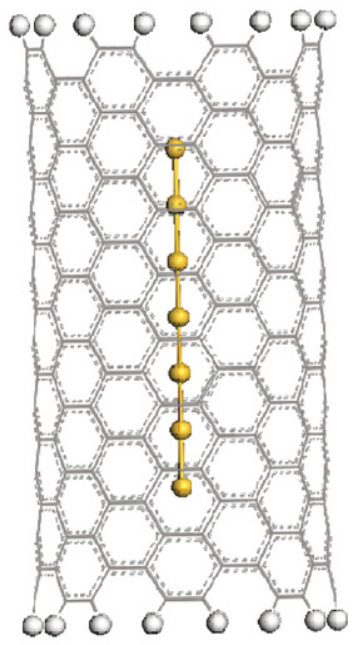

(e)

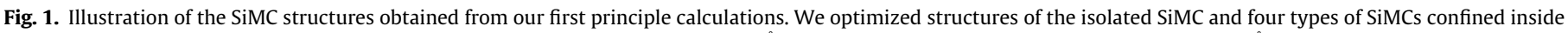
the hydrogenated $(5,5),(6,6),(7,7)$ and $(8,8)$ CNTs. The length of the CNTs is $22.14 \AA$. The radii of the CNTs are $3.39,4.07,4.75$ and $5.43 \AA$, respectively.

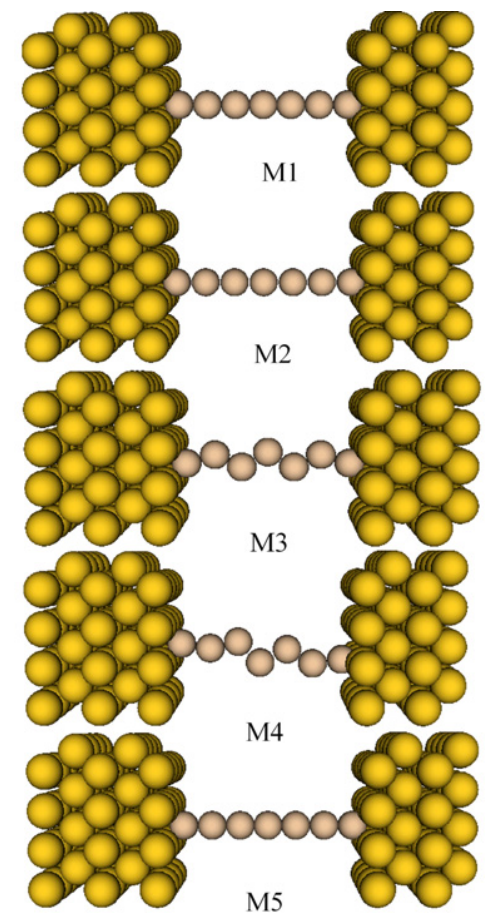

Left electrde! Scattering region , Right electrde

Fig. 2. Schematic description of the two-probe system in our simulations. The isolated and encapsulated SiMCs were sandwiched to two Au $\left(\begin{array}{lll}1 & 0 & 0\end{array}\right)$ electrodes through the Si-Au bond. These five systems were denoted as M1-M5.

results show that bond length and bond angle alterations induced by the encapsulation could enhance the electron transport at high bias voltage. These interesting findings indicate that the SiMC current-voltage characteristics can be regulated by the sizeselective encapsulation. The particular mechanism can be interpreted with the transmission spectrum and spatial distribution of frontier molecular orbitals taken into consideration

\subsection{Low bias conduction}

To explore the current characteristics of the two-probe system, we calculated the transmission coefficient $T(E, V)$ and the

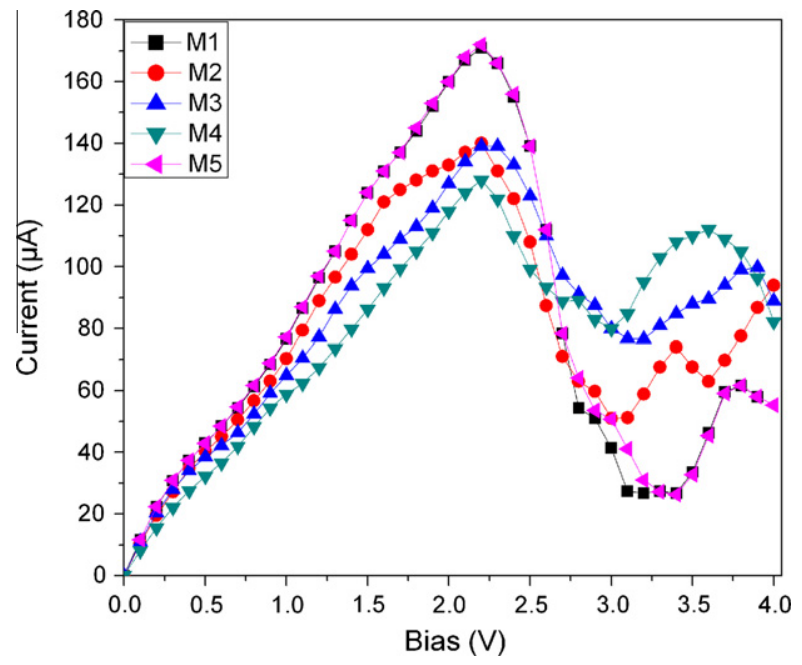

Fig. 3. Calculated currents as a function of the applied bias for systems M1-M5. The NDR behavior can be observed clearly in a certain bias range of $2-4 \mathrm{~V}$.

corresponding frontier molecular orbitals at zero bias with energy window ranging from -2 to $2 \mathrm{eV}$ for systems M1-M5, as shown in Fig. 4a-e. The average Fermi level, which is an average value of the chemical potential of the left and right electrodes, was set as zero. The transmission resonance peak represents the conducting channel. In general, at low applied voltages, it was observed that there are always some transmissions peaks near the Fermi energy resulting in metallic behavior of SiMCs. Under the small bias, the transmission coefficient does not change significantly. If the applied voltage increases, part of the transmission peak around the Fermi level will gradually move into the bias window, giving rise to an initial increase in current for M1-M5. As we know, the electronic transport of the molecular device mostly depends on the frontier molecular orbitals. In Fig. 4a, four molecular orbitals: HOMO (highest occupied molecular orbital), LUMO (lowest unoccupied molecular orbital), LUMO+1, LUMO+2 lie beside the Fermi energy level. This indicates that the electronic transport of M1 at low bias voltage is mainly contributed by the four orbitals. In Fig. 4b, the HOMO shifts obviously to the Fermi energy level while the three LUMOs move far away. Consequently, we can extrapolate 


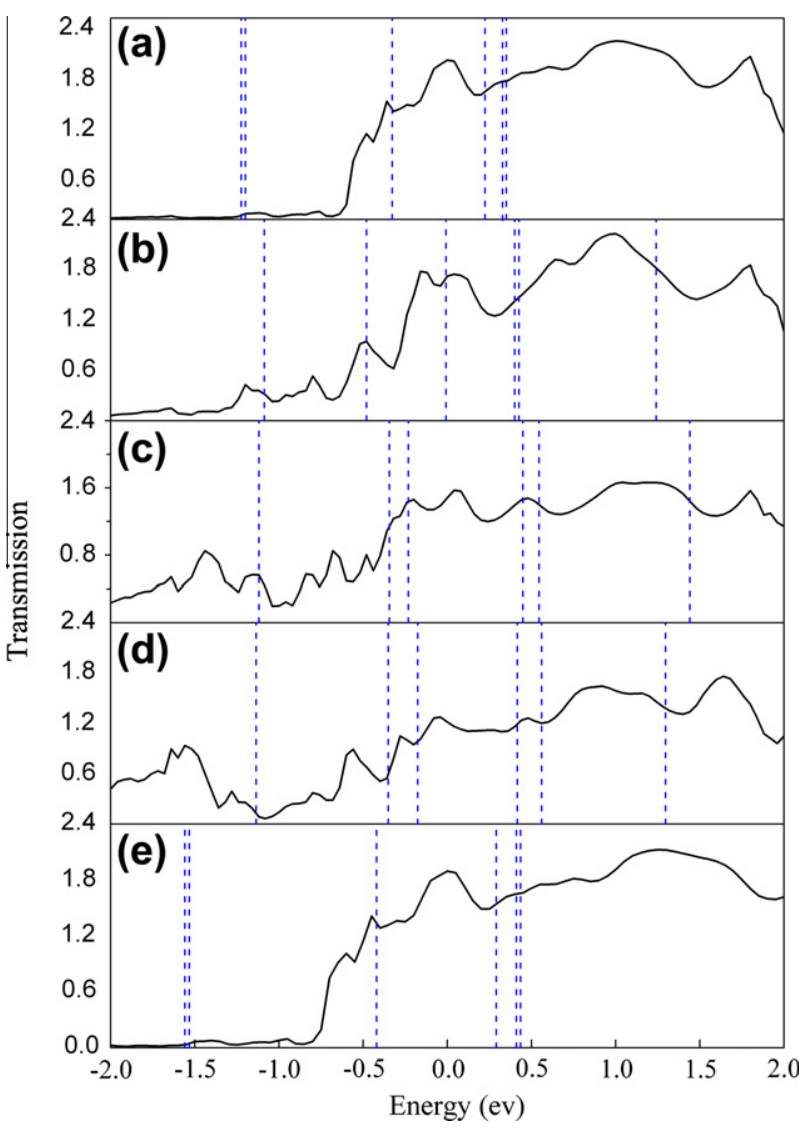

Fig. 4. The transmission spectrum (solid lines) $T(E, V)$ for the two-probe systems M1-M5 shown in Fig. 2 at zero bias. The vertical dashed dotted lines stand for the six frontier molecular orbitals HOMO-2, HOMO-1, HOMO, LUMO, LUMO+1 and LUMO+2, respectively. The average Fermi level was set to be zero.

that the encapsulation induced geometry alteration does affect the energy position of the molecular orbitals. The average transmission coefficient of M2 is smaller than that of M1 which indicates that the electronic transfer ability is reduced when the SiMC is encapsulated. It is known that the transport coefficient is related to the wave function overlap between molecules and electrodes, namely, the coupling degree between molecular orbitals and the incident states from the electrodes. Compared the transmission coefficient of M1 with that of M2, we can find that the electronic transfer ability become weaker resulting from the decrease in the coupling degree between the electrodes and the molecule. In Fig. 4c and d, when the bond angle changes largely due to encapsulation, the HOMO-1, HOMO and LUMO molecular orbitals lie near the Feimi level and mainly contribute to the electronic transport of M3 and M4. It appears that the SiMC reconstruction inside CNTs would reduce the conductance at low bias as reflected by the transmission coefficient. This means that the current can be modulated by the size-selective encapsulation.

\subsection{Negative resistance phenomenon}

In order to understand the NDR behavior in the two probe system, we presented the calculated transmission coefficient.

$T(E, V)$ and the corresponding frontier molecular orbitals HOMO-2, HOMO-1, HOMO, LUMO, LUMO+1, LUMO+2 at special bias voltages of $1.2,2.2,3.0$ and $3.6 \mathrm{~V}$ for M4 in Fig. $5 \mathrm{a}-\mathrm{d}, 3.6 \mathrm{~V}$ for M5 in Fig. 5e, respectively. Eq. (1) indicates that the current is the integral of the transmission coefficient in the bias window $[-V / 2, V / 2]$ and is further determined by the integral area, namely, the shaded area in the bias window as shown in Fig. 5. When the bias is set to be $1.2 \mathrm{~V}$, the bias window is too narrow to include the main transmission channel HOMO-2 and LUMO+1. The large transmission peaks in the energy region between HOMO-2 and HOMO-1, LUMO+1 and LUMO+2 are just lying on the edge of the integral window and do not make contribution to the current as shown in Fig. 5a. Therefore, the currents show small magnitude in this bias region. When the bias takes the value $2.2 \mathrm{~V}$, the bias window is wide enough to include the main transmission waves. The whole transmission peak between HOMO-2 and HOMO-1, and part of the transmission peak between LUMO+1 and LUMO+2 enter into the bias integral window and consequently the total integral area gets lager, as shown in Fig. 5b. As a result, the current increases. However, in Fig. $5 c$ when the external bias voltage is set to be $3.0 \mathrm{~V}$, the bias window increases but the transmission coefficient decreases dramatically. The decrease in the transport coefficient is because of the more wave function overlaps between the molecules and the electrodes, namely, the coupling degree between the molecules orbitals and the electrodes gets weaker with the increase in the bias voltage. Such a decrease may not be compensated by the increase in the bias window, so the integral area gets smaller. As a result, the current decreases and the NDR appears, which has been shown in Fig. 3. When the bias is increased to $3.6 \mathrm{~V}$ in Fig. 5d, a broader transmission valley emerges between -1 and $0.5 \mathrm{eV}$. However, the transmission peak contributed by

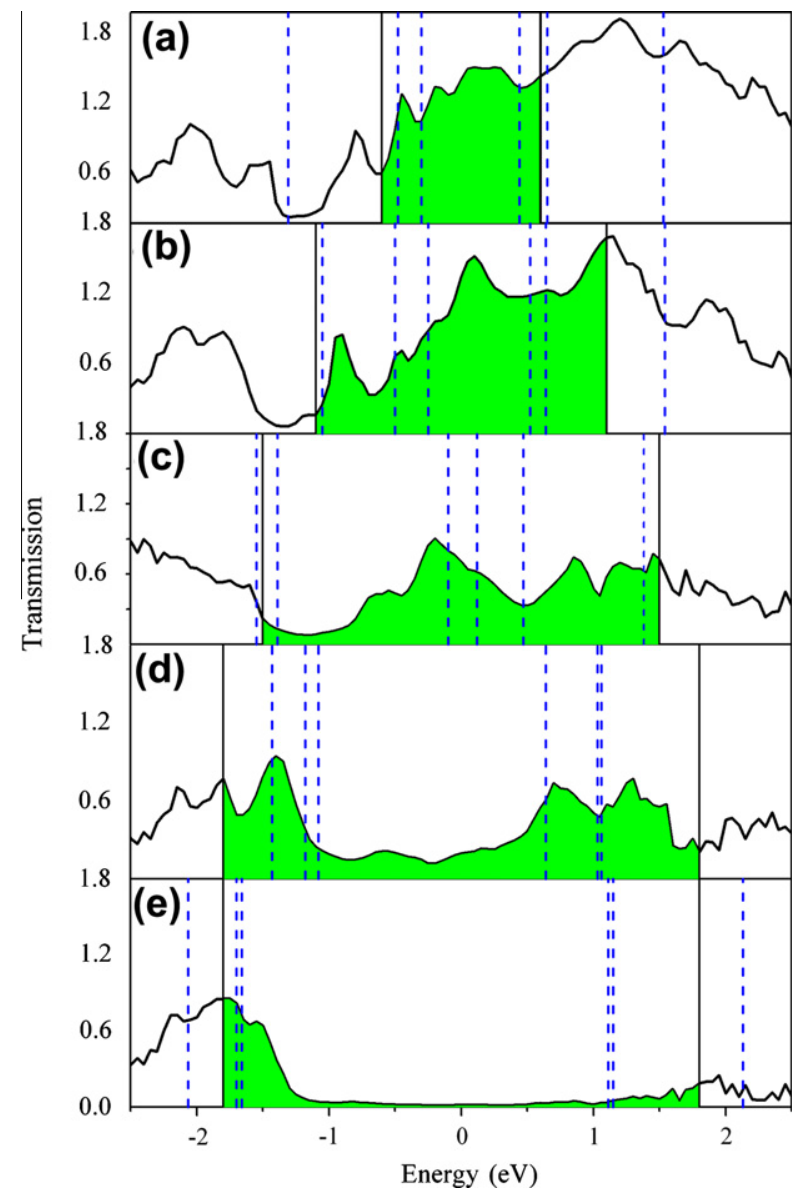

Fig. 5. The transmission coefficient at different bias voltages for the systems M4 and M5. (a)-(d) correspond to the bias voltage being $1.2,2.2,3.0$ and $3.6 \mathrm{~V}$ respectively for M4. (e) corresponds to the bias voltage being $3.6 \mathrm{~V}$ for M5. The region between the solid lines represents the bias window. The shaded area denotes the integral area in the bias window. The dashed lines correspond to the frontier molecular orbitals HOMO-2, HOMO-1, HOMO, LUMO, LUMO+1 and LUMO+2, respectively. 


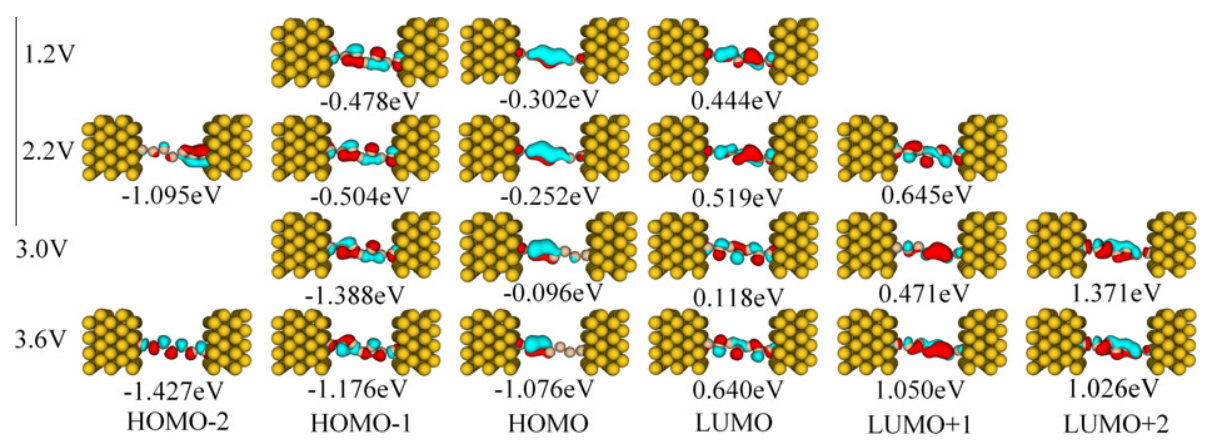

Fig. 6. The MPSH states of the six frontier molecular orbitals HOMO-2, HOMO-1, HOMO, LUMO, LUMO+1 and LUMO+2 for M4 at $1.2 \mathrm{~V}, 2.2 \mathrm{~V}, 3.0 \mathrm{~V}$ and $3.6 \mathrm{~V}$.

HOMO-2 enters into the bias window, thus results in an overall increase of the total integral area and the current increases again. Considering Fig. 5e, a more wide transmission valley with very small transmission coefficient enters the bias window. The current of M5 gets lower than M4 which indicates that the CNTs encapsulation induced SiMC structure variations would enhance the conductance at high bias.

\subsection{The MPSH states}

It has been reported that, in some cases, the NDR behavior originates from the inhibition of the conduction channel at a certain bias [22]. The theoretical work [36] illustrated that the MPSH states of the frontier molecular orbitals localized on any part of the scattering region will not contribute to the transmission spectra and the conduction channel would be suppressed. However, a complete delocalized molecular orbital means that the transmission channel is being opened. Thus it leads to a peak in the transmission spectra. In general, a delocalized molecular orbital contributes more to the transmission probabilities than a localized one in the SiMC system. To evaluate the effect of the localized and delocalized molecular orbitals on the transmission channel, the MPSH and eigenvalues of the frontier molecular orbitals HOMO2, HOMO-1, HOMO, LUMO, LUMO+1 and LUMO+2 were considered in our work. Fig. 6 presented a comparative study of six MPSH states located in the bias window at the bias voltages of 1.2, 2.2, 3.0 and $3.6 \mathrm{~V}$. Comparing the spatial distribution of MPSH states at $1.2 \mathrm{~V}$ and $2.2 \mathrm{~V}$, a complete delocalization can be found in LUMO+1. The delocalized orbital LUMO+1 may form the transmission channel, leading to a big transmission peak. This indicates that the conduction channel LUMO+1 can make a larger contribution to the current when it slipped into the bias window at $2.2 \mathrm{~V}$. Consequently, the current increased as the applied bias voltage in the range of $0-2.2 \mathrm{~V}$, as shown in Fig. 3. When the bias voltage was set to be $3.0 \mathrm{~V}$, the MPSH of the channel HOMO localizes on the left part of the structure, and LUMO+1 distributes significantly on the right side of the molecule. These features clearly indicate that transmission peaks originated from MPSH states HOMO and LUMO+1 will be absent. The coupling degree between the two molecular orbitals and the incident states from the electrodes gets weaker, thus the conduction channels HOMO and LUMO+1 are suppressed. The LUMO+2 is delocalized and opened at the bias voltage of 3.0 V. However, it cannot compensate for the current reduced by the suppressed HOMO and LUMO+1. Consequently, the current at the bias of $3.0 \mathrm{~V}$ is smaller than that at the bias of $2.2 \mathrm{~V}$ and the NDR behavior appears. When the bias is increased to $3.6 \mathrm{~V}$, the channel HOMO-2 is opened, so a transmission peak appears at $E=-1.472 \mathrm{eV}$, as shown in Fig. $5 \mathrm{~d}$. This leads to the remarkable increase of the current. It should be noted that since there are not too many molecular orbitals contributing to the elec- tron transport, the influence of the suppressed channel on the electron current is obvious. Therefore the NDR can be observed. These results also indicate that the decrease of the current shown in Fig. 3 is caused by the suppression of the channel. This explanation can also be use to interpret the NDR behavior in the other systems.

\section{Summary}

In summary, the electric transport properties of the encapsulated SiMCs were investigated using NEGF combined with DFT within a two-probe metal electrode approach. The calculated results showed that the $I-V$ characteristics of SiMCs depend strongly on their geometry structures. The encapsulation induced bond length and bond angle alterations of the SiMC enhance the electron transport at high bias voltage. The NDR behaviors were observed in a certain bias voltage range. Our results indicated that the origin of the NDR behavior includes the variations of the coupling degree between the molecular orbitals and the incident states from the electrodes under different bias voltages, and the conduction channels which are suppressed at a certain bias. The encapsulation induced geometrical variation is thus suggested as an important factor for changing the electronic properties of the SiMCs, which may have potential applications in designing the silicon-based nanoelectronic devices.

\section{Acknowledgment}

This work was supported by the National Natural Science Foundation of China (NSFC, Grant No. 60936001). This work was supported by the National Natural Science Foundation of China (NSFC, Grant Nos. 60936001 and 11021262).

\section{References}

[1] H.S. Park, J.A. Zimmerman, Phys. Rev. B 72 (2005) 054106.

[2] C.Q. Sun, H. Bai, B. Tay, S. Li, E. Jiang, J. Phys. Chem. B 107 (2003) 7544-7546.

[3] A.I. Boukai, Y. Bunimovich, J. Tahir-Kheli, J.K. Yu, W.A. Goddard Iii, J.R. Heath, Nature 451 (2008) 168-171.

[4] E. Garnett, P. Yang, Nano Lett. 10 (2010) 1082-1087.

[5] C.R. Leo, A. Fazzio, A.J.R. da Silva, Nano Lett. 7 (2007) 1172-1177.

[6] S.M. Koo, A. Fujiwara, J.P. Han, E.M. Vogel, C.A. Richter, J.E. Bonevich, Nano Lett. 4 (2004) 2197-2201.

[7] Y. Zhou, X. Zheng, Y. Xu, Z.Y. Zeng, J. Phys. - Condes. Matter 2 (2008) 045225.

[8] J.L. Zang, Q.Z. Yuan, F.C. Wang, Y.P. Zhao, Comput. Mater. Sci. 46 (2009) 621625.

[9] J. Che, T. Cagin, A.G. William III, Nanotechnology 11 (2000) 65-69.

[10] J. Hone, M. Whitney, C. Piskoti, A. Zettl, Phys. Rev. B 59 (1999) 2514-2516.

[11] J.W.G. Wildoer, L.C. Venema, A.G. Rinzler, R.E. Smalley, C. Dekker, Nature 391 (1998) 59-62.

[12] Q.Z. Yuan, Y.P. Zhao, J. Am. Chem. Soc. 131 (2009) 6374-6376.

[13] Y.F. Guo, W.L. Guo, Nanotechnology 17 (2006) 4726.

[14] X. Zhang, H. Li, K. Liew, J. Appl. Phys. 102 (2007) 073709.

[15] X.F. Fan, L. Liu, J.Y. Lin, Z.X. Shen, J.L. Kuo, ACS Nano 3 (2009) 3788-3794.

[16] H. Ohnishi, Y. Kondo, K. Takayanagi, Nature 395 (1998) 780-783. 
[17] Q.Z. Yuan, Y.P. Zhao, P. Roy, Soc. A 468 (2012) 310-322.

[18] B.B. Wang, F.C. Wang, Y.P. Zhao, Sci. China - Phys. Mech. Astron. 55 (2012) $1138-1146$.

[19] M. Bockrath, D.H. Cobden, P.L. McEuen, N.G. Chopra, A. Zettl, A. Thess, R. Smalley, Science 275 (1997) 1922-1925.

[20] G.P. Tang, Z.Q. Fan, X.J. Zhang, Y. Ren, K.Q. Chen, J. Phys. D - Appl. Phys. 42 (2009) 175104

[21] Z.Q. Fan, K.Q. Chen, Q. Wan, B. Zou, W. Duan, Z.G. Shuai, Appl. Phys. Lett. 92 (2008) 263304

[22] X.F. Li, K.Q. Chen, L. Wang, M.Q. Long, B. Zou, Z.G. Shuai, Appl. Phys. Lett. 91 (2007) 133511.

[23] X. Deng, J. Zhou, Z. Zhang, G. Tang M. Qiu, Appl. Phys. Lett. 95 (2009) 103113.

[24] R. Martel, T. Schmidt, H. Shea, T. Hertel, P. Avouris, Appl. Phys. Lett. 73 (1998) 2447-2449.

[25] N.P. Guisinger, M.E. Greene, R. Basu, A.S. Baluch, M.C. Hersam, Nano Lett. 4 (2004) 55-59.

[26] J. Cornil, Y. Karzazi, J. Bredas, J. Am. Chem. Soc. 124 (2002) 3516-3517.
27] J. Taylor, M. Brandbyge, K. Stokbro, Phys. Rev. B 68 (2003) 121101.

[28] M.Q. Long, K.Q. Chen, L. Wang, B. Zou, Z.G. Shuai, Appl. Phys. Lett. 91 (2007) 233512.

[29] J.S. Meisner, M. Kamenetska, M. Krikorian, M.L. Steigerwald, L. Venkataraman, C. Nuckolls, Nano Lett. 11 (2011) 1575-1579.

[30] D.M. Tang, L.C. Yin, F. Li, C. Liu, W.J. Yu, P.X. Hou, B. Wu, Y.H. Lee, X.L. Ma, H.M Cheng, Proc. Natl. Acad. Sci. USA 107 (2010) 9055-9059.

31] M.F. Ng, L. Shen, L. Zhou, S.W. Yang, V.B.C. Tan, Nano Lett. 8 (2008) 3662-3667.

[32] A. Rusznyak, V. Zolyomi, J. Kurti, S. Yang, M. Kertesz, Phys. Rev. B 72 (2005) 155420.

[33] H. Li, X.Q. Zhang, F.W. Sun, Y.F. Li, K.M. Liew, X.Q. He, J. Appl. Phys. 102 (2007) 013702

[34] H. Li, X.Q. Zhang, K.M. Liew, J. Chem. Phys. 128 (2008) 03470307.

[35] M. Brandbyge, J.L. Mozos, P. Ordejon, J. Taylor, K. Stokbro, Phys. Rev. B 65 (2002) 165401.

[36] S. Sen, S. Chakrabarti, J. Phys. Chem. C 112 (2008) 1685-1693. 